\title{
Moving Upstream-Health Extension and Primary Care
}

\author{
Lyle J. Fagnan, MD
}

Health extension programs represent an opportunity for practice-based research networks (PBRNs) and primary care practices to develop collaborations reaching beyond the clinic walls to address the upstream social determinants of health and engage in community-based research. The Health Extension Regional Officers (HEROs) program at the University of New Mexico described in this issue of the JABFM is an innovative model with a bidirectional approach to linking academic health centers to communitybased practices and organizations. Health extension programs are local, influenced by history, relationships, and support. Oregon's health extension workforce represents a diverse group that includes practice facilitators, community health workers, and Cooperative Extension agents. PBRNs are measuring success in terms of collaboration across a spectrum of health activities. The Oregon Rural Practicebased Research Network uses a "Four Pillars" model of community engagement, practice transformation, research, and education to involve researchers, health policy experts, educators, and health extension workers to improve community health. (J Am Board Fam Med 2017;30:10-12.)

The responsibilities and expectations of primary care clinicians and their practices extend beyond the examination room to the community. The practices that I work with in the Oregon Practicebased Research Network (ORPRN) are trying to figure out how to move upstream to address the social determinants of health and keep up with the requirements of the primary care patient-centered medical home. Health extension is a key component of ORPRN's work. Over the past 14 years, the clinicians and practices have developed a view of the network as a resource beyond answering research questions. A clinician-established objective in our bylaws is ". . . conducting research that fosters an understanding of the health care values, dynamics and structure of the practices and their

From the Oregon Rural Practice-based Research Network and the Department of Family Medicine, Oregon Health \& Science University, Portland.

Funding: none.

Conflict of interest: none declared.

Corresponding author: Lyle J. Fagnan, MD, Oregon Health \& Science University, 3181 SW Sam Jackson Park Rd, Mail Code: L222, Portland, OR 97239-3098 (E-mail: fagnanl@ohsu.edu).

\section{See Related Article on page 94.}

communities to improve primary care delivery and community health through sharing and generating evidence-based knowledge." To help meet these objectives, ORPRN ${ }^{1}$ has deployed 9 regionally based practice enhancement research coordinators (PERCs) who bridge the needs of researchers, practices, and the community. Although ORPRN has received funding from a local Clinical and Translational Science Award program, ${ }^{2}$ the Oregon Clinical \& Translational Research Institute, providing funding and time for PERCs to complete activities beyond research studies is a challenge.

The University of New Mexico's (UNM) Health Extension Regional Officer (HERO) Program offers a health extension model to meet these challenges. ${ }^{3}$ HEROs provide an important bidirectional link between academic researchers and the community. The HERO model maintains a focus on community priorities as the education, service, and research resources of the academic health center are extended to community partners. This approach extends the relevance and visibility of the academic health center. The UNM Clinical \& Translational Science Center provides HEROs with training and resources that align with the National Institutes of Health National Center for Advancing Translational 
Sciences goals of building community trust and meeting community needs.

The 2010 Affordable Care Act established the (yet to be funded) Primary Care Extension Program to provide health extension agents to primary care providers to implement and disseminate evidence-based care. Recognizing the promise of the health extension model, the Agency for Healthcare Research and Quality awarded 4 cooperative grants in $2011^{4}$ to test the use of primary care extension agents to implement practice redesign and transformation in small and medium-size independent practices. The project reached academic health centers and the Cooperative Extension Service at land-grant colleges in 18 states. A Health Extension Toolkit ${ }^{5}$ was developed from the experiences of participants. The Agency for Healthcare Research and Quality has extended the health extension model with the EvidenceNOW initiative, where practice facilitators are currently working with 1500 small primary care practices to implement evidence-based cardiovascular preventive care. $^{6}$

Oregon's health extension workforce is a diverse group that includes practice facilitators, community health workers, and Cooperative Extension agents. The 16 coordinated care organizations (CCOs), Oregon's Medicaid accountable care organizations, have embraced the practice facilitation model by deploying staff to work with contracted providers to improve quality metrics. CCOs have also contributed to the development of the community health worker workforce to create a cultur- ally competent bridge between patients and their health and social service providers. Oregon's Cooperative Extension staff, practice facilitators, and community health workers work with the CCOs and state-mandated community advisory councils to ensure that the voice of the community is heard in health transformation efforts.

The Cooperative Extension's National Framework for Health and Wellness ${ }^{7}$ prioritizes making healthy lifestyle choices and educating county residents across the lifespan. The framework has 4 strategic directions: healthy and safe community environments, clinical and community preventive services, empowered people, and elimination of health disparities. The approach used by the Cooperative Extension is built on the Agriculture Extension programs located at land-grant universities to improve and transform agricultural practices. Although most academic health centers do not have strong relationships with Cooperative Extension, the UNM HERO program points to collaboration opportunities for research and improving population health.

ORPRN has developed the "Four Pillars" model to describe our approach to health extension, which includes community organizing, service, research, and education (Figure 1). Health extension is a bridge across the pillars, connecting the ORPRN regional-based PERCs with practices and community stakeholders. Similar to other networks, ${ }^{8}$ ORPRN has become a community and practice resource that includes research but measures its success in terms of meaningful collaborations.

Figure 1. The "four pillars."

Collaborative Community Health: Outreach, Research, Health Transformation, Education

\begin{tabular}{|lll|}
\hline Community \\
Engagement
\end{tabular}


ORPRN has worked to include the community voice in research, and research in community initiatives. This has included transforming the Oregon Community Health Improvement Partnership (CHIP) into the Community Health Improvement and Research Partnership (CHIRP), linking community engagement, research, and education. The CHIP-to-CHIRP program developed and delivered 8 modules to train community groups in how to include research and evaluation in their projects. ${ }^{9,10}$

Although challenging to fund and maintain, the health extension model offers many potential benefits, including increased engagement of communities and stakeholders at all levels; lasting and meaningful research partnerships between academic health centers, practices, and community agencies; and the integration of the numerous efforts to conduct outreach and improve quality of care.

To see this article online, please go to: http://jabfm.org/content/ 30/1/10.full.

\section{References}

1. ORPRN. ORPRN Mission. 2016. Available from: http://www.ohsu.edu/xd/outreach/oregon-ruralpractice-based-research-network/about/our-mission/ index.cfm. Accessed 27 November 2016.

2. National Center for Advancing Translational Sciences, National Institutes of Health. Clinical and Translational Science Awards Program. November 2015. Available from https://ncats.nih.gov/files/ctsa-factsheet.pdf. Accessed November 18, 2016.

3. Kaufman A, Rhyne RL, Anastasoff J, et al. Health extension and clinical and translational science: an innovative strategy for community engagement. J Am Board Fam Med 2017;30:94-9.

4. Agency for Healthcare Research and Quality. IMPaCT (Infrastructure for Maintaining Primary Care Transformation). September 2012. Available from http://www.ahrq.gov/research/findings/factsheets/ primary/impactaw/index.html. Accessed November $18,2016$.

5. Health extension toolkit [homepage on the Internet]. Available from http://healthextensiontoolkit.org/. Accessed November 18, 2016.

6. Cohen DJ, Balasubramanian BA, Gordon L, et al. A national evaluation of a dissemination and implementation initiative to enhance primary care practice capacity and improve cardiovascular disease care: the ESCALATES study protocol. Implement Sci 2016; 11:86.

7. Cooperative Extension's National Framework for Health and Wellness. March 2014. Available from http://www.aplu.org/members/commissions/foodenvironment-and-renewable-resources/CFERR_ Library/national-framework-for-health-andwellness/file?id=5134. Accessed November 18, 2016.

8. Williams RL, Rhyne RL. No longer simply a practice-based research network (PBRN) health improvement networks. J Am Board Fam Med 2011; 24:485-8.

9. Davis MM, Aromaa S, McGinnis PB, et al. Engaging the underserved: a process model to mobilize rural community health coalitions as partners in translational research. Clin Transl Sci 2014;7:300-6.

10. OCTRI. Oregon Clinical \& Translational Research Institute. 2016. OCTRI Research Resources. Available from: http://www.ohsu.edu/xd/research/centersinstitutes/octri/index.cfm. Accessed 27 November, 2016. 